\title{
Experimental Implementation of a Windshield Warning System on an Actual Vehicle*
}

\author{
Chyi-Yeu LIN**, Jinn-Feng JIANG**, Yi-Jie CHEN***, \\ Hung-Yuan WEI*** and Cheng-Hsien WANG** \\ ** Department of Mechanical Engineering, \\ National Taiwan University of Science and Technology, Taipei 10672, Taiwan, R.O.C. \\ *** Project Coordination and Liaison Section, Planning and Promotion Department, \\ Metal Industries Research and Development Center, Taipei 10075, Taiwan, R.O.C. \\ E-mail: yijiechen69@gmail.com
}

\begin{abstract}
A windshield warning system for vehicles is presented. The system assists drivers in identifying potential hazardous object without the need to divert attention from the road. The target is identified by the camera and the software system and then used to construct the line-of-sight equation based on the coordinate system of the moving vehicle. The explicit equation of the intersection point of the line of sight and the windshield surface is derived using an algebraic method. A warning mark is then projected at the intersection point on the windshield by a two-degree-of-freedom laser projector, allowing the driver to easily identify the obstacle. The feasibility of the proposed system was verified using an actual vehicle (Luxgen 7 MPV).
\end{abstract}

Key words: Warning System, Intelligent Vehicle, Driver Assistance

\section{Introduction}

Most traffic accidents are associated with lack of concentration, distraction, or fatigue. Driver assistance systems are designed to assist drivers in identifying obstacles in or near the path of the vehicle.

Several driver-assistance systems have been developed to reduce the number of accidents. Curio et al. proposed an approach for the detection, tracking, and recognition of pedestrians. The initial detection is based on the integration of texture information, template matching, and inverse perspective mapping. After the initial detection, the proposed tracking method is used to recognize pedestrians ${ }^{(1)}$. Stiller et al. developed a multi-sensor system for detecting obstacles and tracking vehicles. The sensor system for obstacle detection comprises a self-assessing vision sensor and a laser scanner. The tracking of obstacles is performed using a Kalman filter whose measurement inputs are the point positions estimated by the vision sensor and a laser scanner ${ }^{(2)}$. Bensrhair et al. presented a cooperative system for vehicle detection that integrates monocular vision and stereo vision to overcome the weaknesses of individual approach ${ }^{(3)}$. Hsieh et al. proposed a vision-based vehicle detection and tracking system for forward collision warning. Various techniques are developed for detection of specific types of obstacle. The processing speed depends on the number of the obstacle candidates ${ }^{(4)}$. Kastrinaki et al. classified the image-processing methods used in traffic applications, and summarized the advantages and disadvantages of each algorithm ${ }^{(5)}$. Huang et al. presented a driving assistance system for locating the positions of lane boundaries and for detecting vehicles in the front. A Gaussian filter, a

Received 29 May, 2012 (No. 12-0236) [DOI: 10.1299/jamdsm.6.1250]

Copyright () 2012 by JSME peak-finding procedure, and a line-segment grouping procedure are used for lane 
recognition and vehicle detection ${ }^{(6)}$. Li et al. proposed a moving-vehicle detection and tracking system for real-time operation. The system consists of three components: road detection, vehicle detection, and vehicle tracking ${ }^{(7)}$. McCall and Trivedi discussed various lane-detection and position-estimation algorithms. They also presented the video-based lane estimation and tracking system for driver assistance, and conducted experiments on an instrumented-vehicle test bed ${ }^{(8)}$.

Wei et al. presented a vehicle frontal collision warning system based on target tracking and threat assessment for collision avoidance. The longitudinal and lateral position of objects obtained from a radar and a lidar, and the host's speed and angular velocity are used to track targets in the front of the vehicle ${ }^{(9)}$. Sengupta et al. proposed a cooperative collision warning system. The system provides warnings or situation awareness to drivers based on information about the motions of neighboring vehicles obtained via the wireless communication with those vehicles, without use of any ranging sensors. The system is inexpensive compared to those that use ranging sensors with a 360 -degree coverage ${ }^{(10)}$. Jenkins et al. developed a warning system to reduce the likelihood of a lateral collision. The problem was analyzed using the work domain analysis, and the results provided information useful for designing an interface for users ${ }^{(11)}$. Hammoud et al. proposed a driver distraction alert system. They integrated the lane departure warning (LDW) and the forward collision warning systems ${ }^{(12)}$. Kim et al. developed an all-around sensing system based on an integrated advanced driver-assistance system that senses in all directions using two cameras and eight sonar sensors. The system recognizes the driving environment via the lane and vehicle detection ${ }^{(13)}$.

Recently, Gao et al. presented a practical algorithm for road detections based on computer vision which implements the lane recognition and vehicle techniques ${ }^{(14)}$. Hsiao et al. developed an embedded real-time LDW system. The lane detection rate of the system is $99.57 \%$ during the day and $98.88 \%$ at night in a highway environment. The proposed LDW system effectively generates warning signals and gives few false warnings ${ }^{(15)}$. Lin et al. presented a robust lane detection system constructed with a lateral inhibition and a conjugate Gaussian model. The system uses two- and three-dimensional geometric information to verify lane marks ${ }^{(16)}$. Lin and Li proposed a field programmable gate array (FPGA) architecture and implementation for a blind-spot warning system. This system uses only $13 \%$ logic resources and $95 \mathrm{k}$-bits of the FPGA's block memory, and its frame rate is over 30 frames per second ${ }^{(17)}$. Flores et al. presented an automatic driver drowsiness detection system based on visual information and artificial intelligence. The system tracks and analyzes the face and the eyes to compute a drowsiness index. The algorithm was validated in a real vehicle ${ }^{(18)}$. Dahmani et al. developed a lane departure detection method and simulated it using the full vehicle dynamics software CarSim ${ }^{\oplus}$. The proposed algorithm uses steering dynamics and time information to the lane keeping system to reduce the number of false alarms. Simulation results showed that the method had good efficiency under various driving scenarios ${ }^{(19)}$.

Most driver-assistance systems require the driver to divert attention from the road to see real-time auxiliary warning information on a monitor, which is very dangerous during driving. The present study proposes a windshield warning system in which a two-degree-of-freedom laser projector is used to display information directly on the windshield. The remainder of this paper is organized as follows. The concept of windshield warning system and its mathematical model are discussed in Section 2. Section 3 describes the experiment and the vehicle verification platform used for evaluation of the system in an outdoor environment. In Section 4, the results of experiments are discussed. Finally, Section 5 presents the conclusions and discusses future research. 


\section{Nomenclature}

${ }^{0} A_{1},{ }^{0} A_{2}$

${ }^{0} \mathbf{D},{ }^{0} \mathbf{P}$

${ }^{0} \mathbf{I},{ }^{3} \mathbf{I}$

${ }^{1} \mathbf{D},{ }^{2} \mathbf{O}$

${ }^{3} A_{0}$

${ }^{3} x_{i},{ }^{3} y_{i},{ }^{3} z_{i}$

$D$

$O$

$a_{w}$

$\mathbf{e}_{1}, \mathbf{e}_{2}$

$\mathbf{e}_{3}$

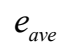

$e_{\text {ave,trial }}$

$e_{k}$

n

$\mathbf{n}_{1}, \mathbf{n}_{2}, \mathbf{n}_{3}$

$n$

$p_{x}, p_{y}, p_{z}$

$q_{x}, q_{y}, q_{z}$

$\mathbf{r}_{O D}$

$s_{x}, s_{y}, s_{z}$

$\mathbf{u}_{x}, \mathbf{u}_{y}$

$v$

$v_{1}, v_{2}, v_{3}$

$\mathbf{x}_{\mathbf{j}}$

$\mathbf{X}_{w}$

$x_{0}-y_{0}-z_{0}$

$x_{1}-y_{1}-z_{1}$

$x_{2}-y_{2}-z_{2}$

$x_{3}-y_{3}-z_{3}$

$x_{D}, y_{D}, z_{D}$
The transformation matrix of the $x_{1}-y_{1}-z_{1}$ and the $x_{2}-y_{2}-z_{2}$ coordinate system with respect to $x_{0}-y_{0}-z_{0}$ coordinate system, respectively.

The position vectors of the obstacle and driver with respect to the $x_{0}-y_{0}-z_{0}$ coordinate system, respectively, $\mathrm{cm}$.

The position vectors of the intersection point $I$ with respect to the $x_{0}-y_{0}-z_{0}$ and $x_{3}-y_{3}-z_{3}$ coordinate systems, respectively, $\mathrm{cm}$.

The position vectors of the driver and the obstacle with respect to the $x_{1}-y_{1}-z_{1}$ and $x_{2}-y_{2}-z_{2}$ coordinate systems, respectively, $\mathrm{cm}$.

The transformation matrix of the $x_{0}-y_{0}-z_{0}$ coordinate system with respect to the $x_{3}-y_{3}-z_{3}$ coordinate system.

The coordinates of the intersection point on the windshield with respect to the $x_{3}-y_{3}-z_{3}$ coordinate system, $\mathrm{cm}$.

The center of the two eyes of the driver.

The center of an obstacle in front of the vehicle.

The distance in the $x$-direction between the windshield and the fixed coordinate system, $\mathrm{cm}$.

The independent planes used for describing the line of sight.

The independent plane used for describing the windshield of the vehicle.

The average position error for all trials, $\mathrm{cm}$.

The average position error for a trial, $\mathrm{cm}$.

The position error for a screenshot, $\mathrm{cm}$.

The normal vector of a general plane; the $x, y, z$ components of the normal vector are referred to as $m, n, u$, respectively, $\mathrm{cm}$.

The normal vectors of planes $\mathbf{e}_{1}, \mathbf{e}_{2}$, and $\mathbf{e}_{3}$, respectively, $\mathrm{cm}$.

The number of experiments.

The coordinates of the origin of the $x_{1}-y_{1}-z_{1}$ coordinate system relative to the $x_{0}-y_{0}-z_{0}$ coordinate system, $\mathrm{cm}$.

The coordinates of the origin of the $x_{2}-y_{2}-z_{2}$ coordinate system relative to the $x_{0}-y_{0}-z_{0}$ coordinate system, $\mathrm{cm}$.

The position vector between the driver and an obstacle, $\mathrm{cm}$.

The coordinates of the origin of the $x_{3}-y_{3}-z_{3}$ coordinate system relative to the $x_{0}-y_{0}-z_{0}$ coordinate system, $\mathrm{cm}$.

The unit vectors in the $X$ - and $Y$-direction of the $x_{0}-y_{0}-z_{0}$ coordinate system, respectively.

The coefficient of a general plane equation.

The coefficients of planes $\mathbf{e}_{1}, \mathbf{e}_{2}$, and $\mathbf{e}_{3}$, respectively.

The vector of a point on the general plane; the $x, y, z$ components

of this vector are referred to as $x_{j}, y_{j}, z_{j}$, respectively, $\mathrm{cm}$.

The position vector of a point on plane $\mathbf{e}_{3}, \mathrm{~cm}$.

The fixed coordinate system of the windshield warning system.

The camera coordinate system for the face of the driver.

The camera coordinate system for the obstacle.

The coordinate system of the laser projector unit.

The coordinates of the driver with respect to the fixed coordinate system, $\mathrm{cm}$. 


\begin{tabular}{|c|c|}
\hline$x_{e, k}, y_{e, k}$ & $\begin{array}{l}\text { The distances in the } X \text { - and } Y \text {-directions between the warning } \\
\text { frame and the center of the obstacle, respectively, } \mathrm{cm} \text {. }\end{array}$ \\
\hline$x_{i}, y_{i}, z_{i}$ & $\begin{array}{l}\text { The coordinates of the intersection point on the windshield with } \\
\text { respect to the fixed coordinate system, } \mathrm{cm} \text {. }\end{array}$ \\
\hline$x_{O}, y_{O}, z_{O}$ & $\begin{array}{l}\text { The coordinates of the obstacle with respect to the fixed } \\
\text { coordinate system, } \mathrm{cm} \text {. }\end{array}$ \\
\hline$\theta_{p}, \quad \theta_{t}$ & The pan and tilt angles of the laser projector, respectively, degree. \\
\hline$\theta_{w}$ & $\begin{array}{l}\text { The angle of inclination of the windshield with respect to the } \\
\text { horizontal plane, degree. }\end{array}$ \\
\hline
\end{tabular}

\section{Mathematical model of proposed windshield warning system}

The concept of the proposed windshield warning system is shown in Fig. 1. The system is designed to display warnings directly on the windshield of the vehicle. If an object obtained by the camera system is predicted to be hazardous by the software system, the coordinates of the obstacle relative to the moving vehicle are calculated in real-time. The line-of-sight equation is constructed from the coordinates of the eyes of the driver and the hazardous obstacle. The intersection point of the line of sight and the windshield surface is calculated using an algebraic method. Then, a warning mark is projected at the intersection point on the windshield by a laser projector. A verification platform was constructed for an actual vehicle to demonstrate the proposed system. The proposed windshield warning system helps drivers identify potential hazardous obstacle in the front without the need to divert attention away from the road.

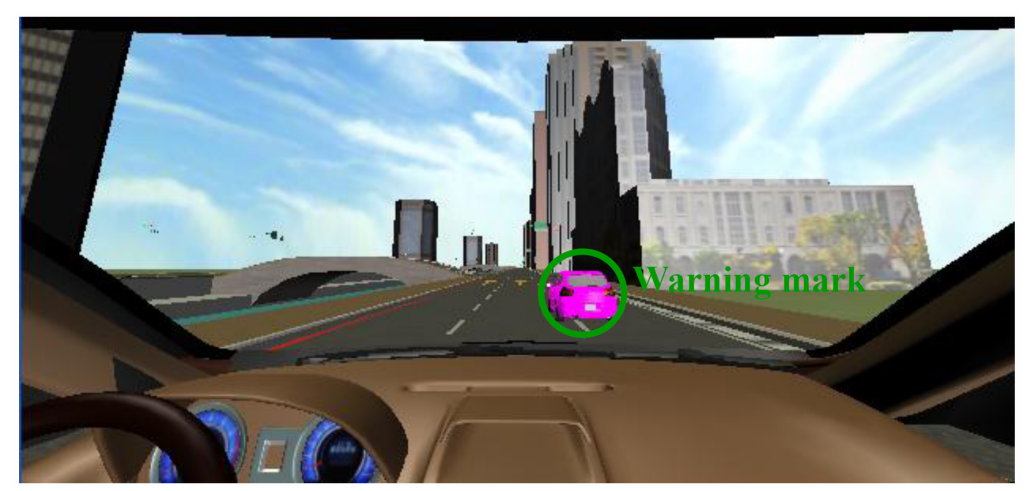

Fig. 1. Proposed windshield warning system.

In the proposed system, the coordinates of the eyes of the driver and the potential hazardous obstacle relative to the moving vehicle are obtained using image recognition techniques. Using an algebraic method, the explicit equation of the intersection point $I$ of the line-of-sight and the windshield surface is derived.

The coordinate systems of the windshield warning system are shown in Fig. 2. $x_{0}-y_{0}-z_{0}$ indicates the coordinate system of the steering wheel and is used as the fixed coordinate system of the windshield warning system. $x_{1}-y_{1}-z_{1}$ and $x_{2}-y_{2}-z_{2}$ are the face (center of two eyes) of the driver and the camera coordinate systems for the obstacle, respectively. $x_{0}-y_{0}-z_{0}$ indicates the coordinate system of the laser projector unit. ${ }^{1} \mathbf{D}$ and ${ }^{2} \mathbf{O}$ are the position vectors of the driver and obstacle with respect to the $x_{1}-y_{1}-z_{1}$ and $x_{2}-y_{2}-z_{2}$ coordinate systems, respectively.

Consider position vectors ${ }^{1} \mathbf{D}$ and ${ }^{2} \mathbf{O}$ with respect to the $x_{0}-y_{0}-z_{0}$ coordinate system. Using the transformation matrix for a pure translation, position vectors ${ }^{0} \mathbf{D}$ and ${ }^{0} \mathbf{O}$ can be expressed as: 


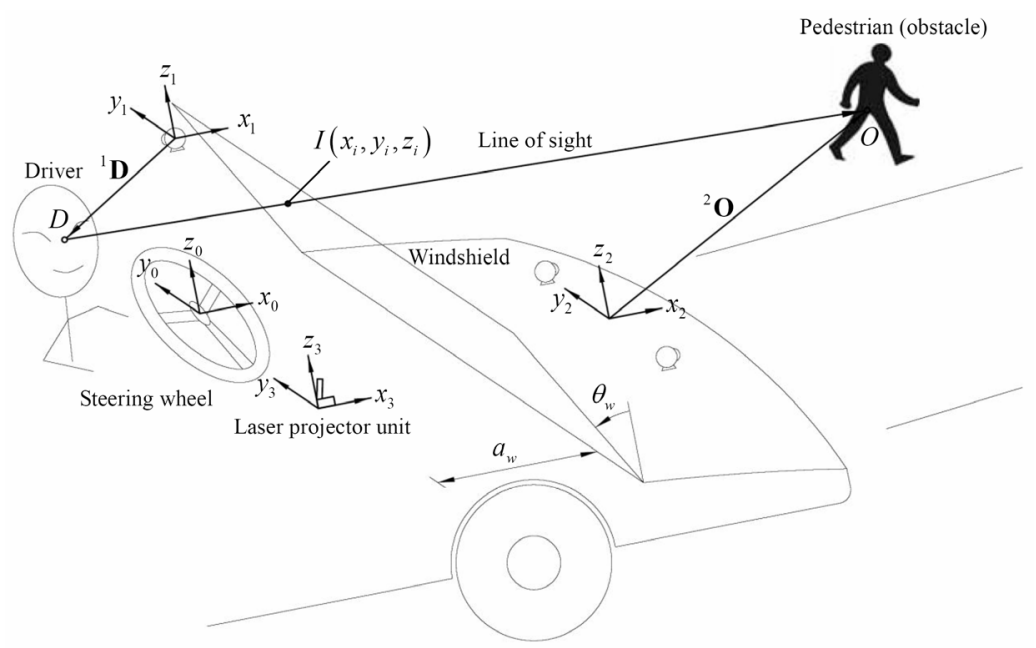

Fig. 2. Coordinate systems of the proposed system.

$$
\begin{gathered}
{ }^{0} \mathbf{D}=\left[x_{D}, y_{D}, z_{D}, 1\right]^{T}={ }^{0} A_{1}^{1} \mathbf{D} \\
{ }^{0} \mathbf{O}=\left[x_{O}, y_{O}, z_{O}, 1\right]^{T}={ }^{0} A_{2}^{2} \mathbf{O}
\end{gathered}
$$

where ${ }^{0} A_{1}=\left[\begin{array}{cccc}1 & 0 & 0 & p_{x} \\ 0 & 1 & 0 & p_{y} \\ 0 & 0 & 1 & p_{z} \\ 0 & 0 & 0 & 1\end{array}\right]$ and ${ }^{0} A_{2}=\left[\begin{array}{cccc}1 & 0 & 0 & q_{x} \\ 0 & 1 & 0 & q_{y} \\ 0 & 0 & 1 & q_{z} \\ 0 & 0 & 0 & 1\end{array}\right]$.

The line of sight can be constructed using ${ }^{0} \mathbf{D}$ and ${ }^{0} \mathbf{O}$. Two independent planes can describe a line ${ }^{(20)}$. Therefore, two independent planes, $\mathbf{e}_{1}$ and $\mathbf{e}_{2}$, are created to describe the line of sight for the proposed system.

For the algebraic plane shown in Fig. $3^{(21)}$, the equation of a plane with nonzero normal vector $\mathbf{n}=(a, b, c)$ through the point $\mathbf{x}_{\mathbf{j}}=\left(x_{j}, y_{j}, z_{j}\right)$ is:

$$
\mathbf{n} \cdot\left(\mathbf{x}-\mathbf{x}_{\mathbf{j}}\right)=0
$$

where $\mathbf{x}=(x, y, z)$. Substituting the values gives the general equation of a plane:

$$
m x+n y+u z+v=0
$$

where $v=-m x_{j}-n y_{j}-u z_{j}$.

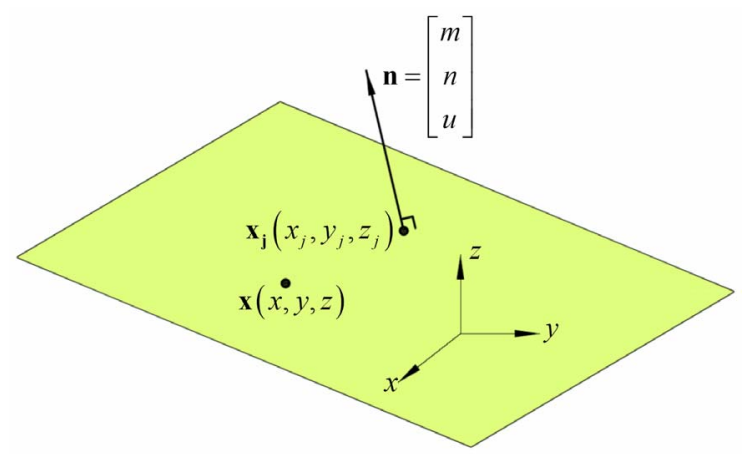

Fig. 3. Algebraic plane. 
Accordingly, the two independent planes $\mathbf{e}_{1}$ and $\mathbf{e}_{2}$ are established as shown in Fig. 4. The normal vector $\mathbf{n}_{1}$ of plane $\mathbf{e}_{1}$ can be obtained from the cross product of $\mathbf{r}_{O D}$ and $\mathbf{u}_{x}$ :

$$
\mathbf{n}_{1}=\mathbf{r}_{O D} \times \mathbf{u}_{x}
$$

where $\mathbf{r}_{O D}={ }^{0} \mathbf{O}-{ }^{0} \mathbf{D}$ is the position vector between the driver and an obstacle, and $\mathbf{u}_{x}$ is a unit vector in the $x$-direction of the $x_{0}-y_{0}-z_{0}$ coordinate system. Similarly, the normal vector $\mathbf{n}_{2}$ of plane $\mathbf{e}_{2}$ can be written as:

$$
\mathbf{n}_{2}=\mathbf{r}_{O D} \times \mathbf{u}_{y}
$$

where $\mathbf{u}_{y}$ is a unit vector in the $y$-direction of the $x_{0}-y_{0}-z_{0}$ coordinate system.

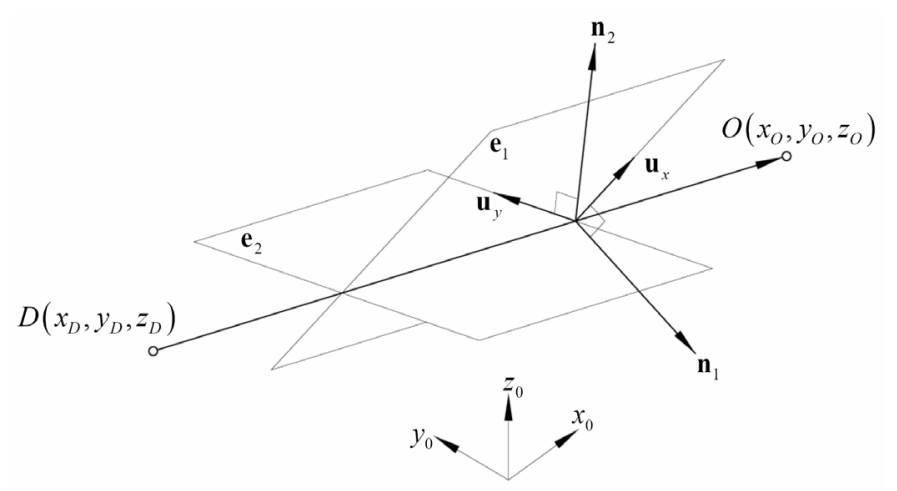

Fig. 4. Geometric relationship of planes $\mathbf{e}_{1}$ and $\mathbf{e}_{2}$.

Point ${ }^{0} \mathbf{O}\left(x_{O}, y_{O}, z_{O}\right)$ is located on planes $\mathbf{e}_{1}$ and $\mathbf{e}_{2}$. Substituting $\mathbf{n}_{1}$ and ${ }^{0} \mathbf{O}$ into Eq. (4), the coefficient $v_{1}$ can be solved as:

$$
v_{1}=-y_{O} z_{D}+y_{D} z_{O}
$$

Similarly, substituting $\mathbf{n}_{2}$ and ${ }^{0} \mathbf{O}$ into Eq. (4), the coefficient $v_{2}$ can be also solved as:

$$
v_{2}=x_{P} z_{D}-x_{D} z_{P}
$$

Then, the equations of planes $\mathbf{e}_{1}$ and $\mathbf{e}_{2}$ with respect to the $x_{0}-y_{0}-z_{0}$ coordinate system are respectively obtained as:

$$
\begin{aligned}
& \mathbf{e}_{1}:\left(-z_{D}+z_{O}\right) y+\left(y_{D}-y_{O}\right) z+\left(y_{O} z_{D}-y_{D} z_{O}\right)=0, \quad x \in R \\
& \mathbf{e}_{2}:\left(z_{D}-z_{O}\right) x+\left(-x_{D}+x_{O}\right) z+\left(x_{D} z_{O}-x_{O} z_{D}\right)=0, \quad y \in R
\end{aligned}
$$

The independent planes $\mathbf{e}_{1}$ and $\mathbf{e}_{2}$ describe the line of sight for the proposed system.

An actual automobile windshield has a complex geometry. For simplicity, the complex surface of an actual windshield is modeled as a plane. This plane, $\mathbf{e}_{3}$, is defined as a virtual windshield. From the coordinates of the windshield warning system shown in Fig. 2, the normal vector $\mathbf{n}_{3}$ of plane $\mathbf{e}_{3}$ can be expressed as:

$$
\mathbf{n}_{3}=\left(\cos \theta_{w}, 0, \sin \theta_{w}\right)
$$

A point $\mathbf{x}_{w}\left(a_{w}, 0,0\right)$ is located on plane $\mathbf{e}_{3}$. Substituting $\mathbf{n}_{3}$ and $\mathbf{x}_{w}$ into Eq. (4), the 
coefficient $v_{3}$ can be solved as:

$$
v_{3}=-a_{w} \cos \theta_{w}
$$

Hence, the equation of plane $\mathbf{e}_{3}$ with respect to the $x_{0}-y_{0}-z_{0}$ coordinate system can be written as:

$$
\mathbf{e}_{3}: x \cos \theta_{w}+z \sin \theta_{w}-a_{w} \cos \theta_{w}=0, \quad z \in R
$$

Using the basic geometry concept that independent planes $\mathbf{e}_{1}, \mathbf{e}_{2}$, and $\mathbf{e}_{3}$ intersect at a point, the explicit equations of intersection point $I$ on the windshield can be obtained as:

$$
\begin{gathered}
x_{i}=\frac{a_{w}\left(x_{D}-x_{O}\right) \cos \theta_{w}+\left(x_{O} z_{D}-x_{D} z_{O}\right) \sin \theta_{w}}{\left(x_{D}-x_{O}\right) \cos \theta_{w}+\left(z_{D}-z_{O}\right) \sin \theta_{w}} \\
y_{i}=\frac{\left(-x_{O} y_{D}+a_{w}\left(y_{D}-y_{O}\right)+x_{D} y_{O}\right) \cos \theta_{w}+\left(y_{O} z_{D}-y_{D} z_{O}\right) \sin \theta_{w}}{\left(x_{D}-x_{O}\right) \cos \theta_{w}+\left(z_{D}-z_{O}\right) \sin \theta_{w}} \\
z_{i}=\frac{\left(-x_{O} z_{D}+a_{w}\left(z_{D}-z_{O}\right)+x_{D} z_{O}\right) \cos \theta_{w}}{\left(x_{D}-x_{O}\right) \cos \theta_{w}+\left(z_{D}-z_{O}\right) \sin \theta_{w}}
\end{gathered}
$$

After the position of intersection point $I$ has been found using Eqs. (14)-(16), an industrial PC commands the two servo motors to rotate to the specified direction so that the laser projector can emit a beam to intersection point $I$. The required rotation angles of servo motors $\# 1$ and $\# 2$ are derived as follows. As shown in Fig. 5, using the transformation matrix for a pure translation, the position vector ${ }^{3} \mathbf{I}$ can be written as:

$$
{ }^{3} \mathbf{I}=\left[{ }^{3} x_{i},{ }^{3} y_{i},{ }^{3} z_{i}, 1\right]^{T}={ }^{3} A_{0}{ }^{0} \mathbf{I}
$$

where ${ }^{3} A_{0}=\left[\begin{array}{cccc}1 & 0 & 0 & s_{x} \\ 0 & 1 & 0 & s_{y} \\ 0 & 0 & 1 & s_{z} \\ 0 & 0 & 0 & 1\end{array}\right]$ and ${ }^{0} \mathbf{I}=\left[x_{i}, y_{i}, z_{i}, 1\right]^{T}$.

Using a trigonometric identity, the rotation angles $\theta_{p}$ and $\theta_{t}$ of servo motors \#1 and \#2 can be respectively obtained as:

$$
\begin{gathered}
\theta_{p}=\tan ^{-1}\left({ }^{3} y_{i} /{ }^{3} x_{i}\right) \\
\theta_{t}=\tan ^{-1}\left(\left({ }^{3} z_{i}-s_{z}\right) / \sqrt{\left({ }^{3} x_{i}\right)^{2}+\left({ }^{3} y_{i}\right)^{2}}\right)
\end{gathered}
$$

\section{Windshield warning system and verification platform}

Based on the mathematical model of the windshield warning system, a verification platform was constructed on an actual vehicle to verify the function of the proposed system. A flow chart of the verification platform is shown in Fig. 6. 


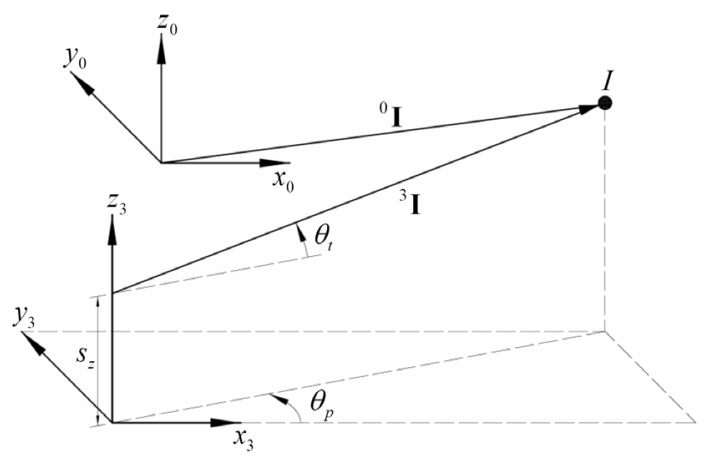

Fig. 5. Relationship between servo motor angles $\theta_{p}$ and $\theta_{t}$.

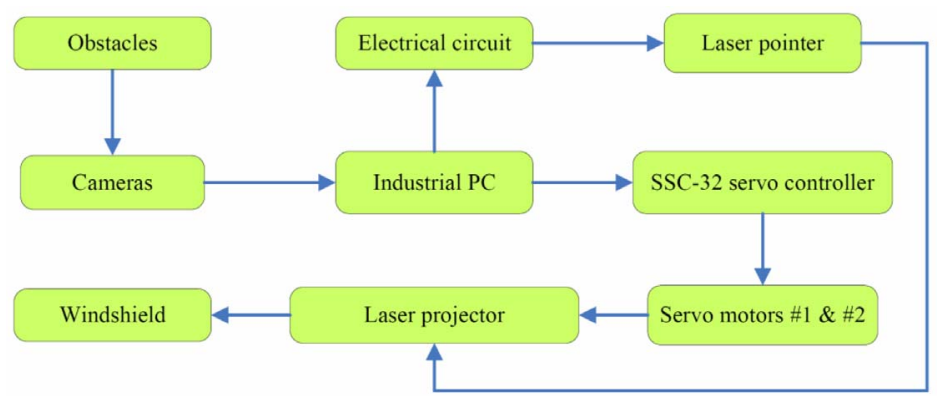

Fig. 6. Flow chart of verification platform for proposed system.

A Luxgen ${ }^{\circledast} 7 \mathrm{MPV}^{(22)}$, as shown in Fig. 7, was used as the experiment platform. Two cameras are used to capture the frontal images so as to predict the hazardous obstacles. After identification of an obstacle, the coordinates of the obstacle relative to the moving vehicle are calculated using image processing software in the industrial PC. The industrial PC also controls the motion of the two servo motors of the laser projector unit to emit the laser beam to form a mark at the right position of the windshield for driver to easily track the obstacle. The industrial PC is an embedded device with a 1.0-GHz dual-core CPU (see Fig. 8$)^{(23)}$. To enable a real-time operation, the system was implemented in $\mathrm{C}$ programming language.
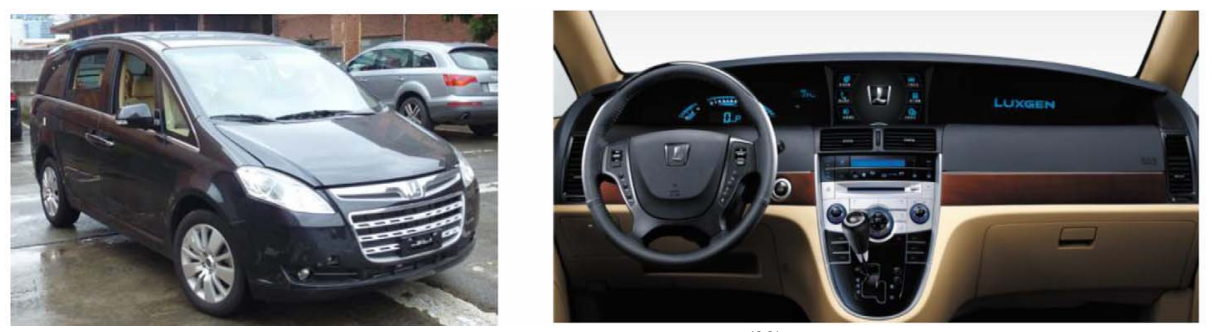

Fig. 7. Luxgen ${ }^{\circledR} \mathrm{MPV}^{(22)}$.

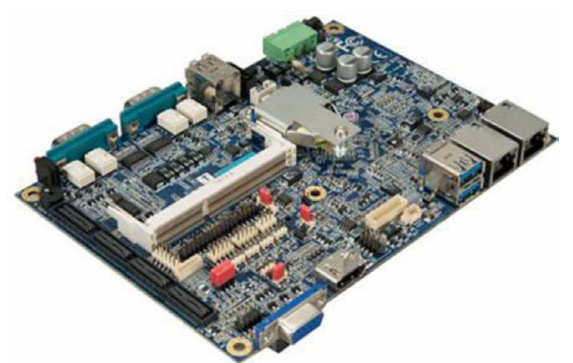

Fig. 8. Industrial PC EITX-3002(23). 
The industrial PC communicates with the laser pointer and two servo motors over the recommended standard 232 (RS-232) interface. The laser projector is moved by two servo motors (S3102, Futaba Company). The dimensions and specifications of the S3102 servo motor are shown in Fig. 9. A servo controller (SSC-32, Lynxmotion Company), shown in Fig. 10, is used to control the motions of the two servo motors on the laser projector. The industrial PC and laser projector use $12-\mathrm{V}$ power supplies, respectively.

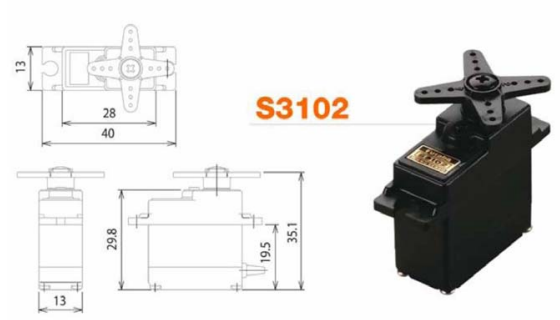

\begin{tabular}{c|c}
\hline Type & Analog Micro \\
\hline Speed & $0.2 \mathrm{~s} / 60^{\circ} @ 6 \mathrm{~V}$ \\
\hline Torque & $4.6 \mathrm{~kg} / \mathrm{cm} @ 6 \mathrm{~V}$ \\
\hline Dimensions & $28 \mathrm{~mm} \times 13 \mathrm{~mm} \times 30 \mathrm{~mm}$ \\
\hline Weight & $21 \mathrm{~g}$ \\
\hline Bearings & Bronze \\
\hline Gear type & Metal \\
\hline \hline
\end{tabular}

Fig. 9. Futaba S3102 servo motor ${ }^{(24)}$.

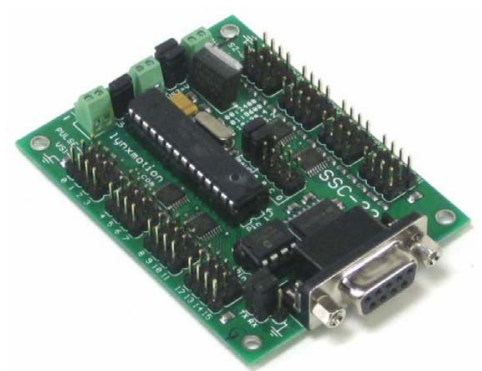

Fig. 10. SSC-32 servo controller ${ }^{(25)}$.

Control software is used for the laser pointer and servo motors. The interface of the control software for the laser projector was developed in Borland ${ }^{\circledR} \mathrm{C}++$ Builder 6.0. The control software is used to test the characteristics of the laser projector, including limit positions, speeds, and calibration data. These characteristics are considered in the control program to increase the accuracy of laser projector control.

A warning mark at the intersection point on the windshield is displayed by the laser projector unit. The motion of the laser projector unit is two-dimensional, and thus requires two actuators, each providing one-dimensional independent motion. The structure of the laser projector was designed using the Solidworks ${ }^{\circledR} 2008$.

The vehicle experiment platform of the proposed windshield warning system is shown in Fig. 11(a). The warning frame is a green point on the windshield as shown in Fig. 11(b), and the black paper on the windshield is used to increase the brightness of the warning frame. The experimental trials were recorded using a camera, which was located where the driver's eyes would be.

\section{Experimental Results and Discussion}

The verification platform was constructed on an actual vehicle to demonstrate the feasibility of the proposed real time windshield warning system. Figure 12 shows a photograph of the actual experiment, where the green point is the center of the warning frame, and the white cross indicates the center of the obstacle (a security booth). In ideal conditions, the warning frame projected by the laser projector should coincide with the center of the obstacle. However, due to mechanical assembly errors, a delayed response time, noise interference, and other factors, there is a discrepancy between the center of the warning frame and the center of the obstacle. Since the output of the system is a warning frame displayed on the windshield, the discrepancy (position error) between the center of 
the warning frame and the center of the obstacle is the simplest and most direct performance index.

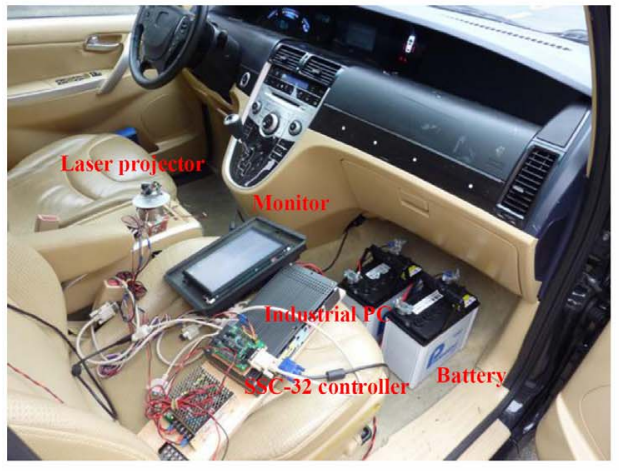

(a) Experiment platform.

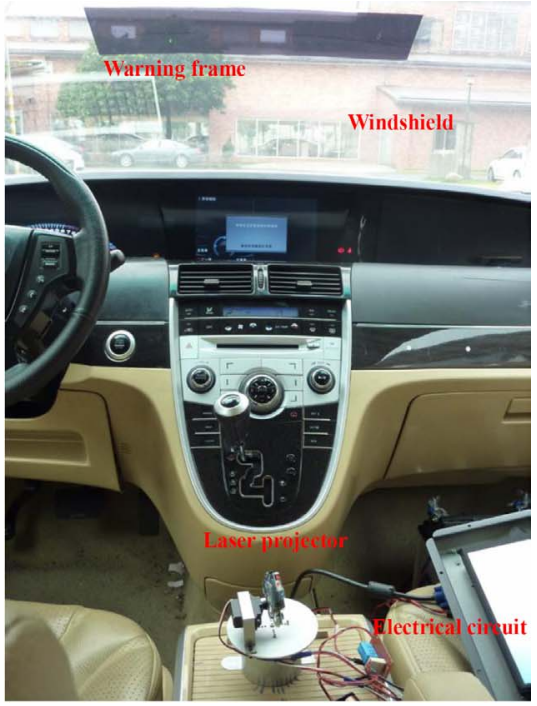

(b) Warning frame.

Fig. 11. Vehicle experiment platform.

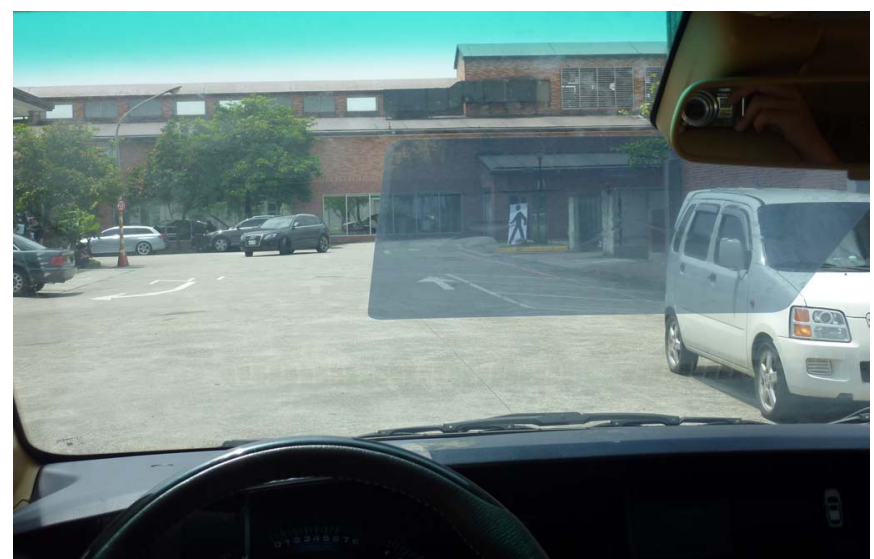

Fig. 12. Experiment for proposed warning system.

The experiment output, including the displayed film clip and the real-time laser projection, was recorded using a camera. After the experiment, screenshots of the recorded movie were captured every time step $\left(t_{d}\right)$ using a video processing software. The position error $e_{i}$ for each screenshot was calculated as:

$$
e_{k}=\sqrt{x_{e, k}^{2}+y_{e, k}^{2}}, k=1,2,3, \ldots, N
$$

where $x_{e}$ and $y_{e}$ are the distances in the $x$-and $y$-directions between the center of the warning frame and the center of the obstacle, respectively. $N$ is the number of screenshots taken during the experiment. Hence, the average position error for each trial $e_{\text {ave,trial }}$ was calculated as the sum of the position error for each screenshot divided by the total number of screenshots in the trial:

$$
e_{\text {ave }, \text { trial }}=\frac{\sum_{k=1}^{N} e_{k}}{N}
$$




$$
N=\frac{t_{f}-t_{i}}{t_{d}}
$$

where $t_{i}$ is the initial time, $t_{f}$ is the final time, and $t_{d}$ is the time step. The average position error for $n$ experiments can be expressed as:

$$
e_{\text {ave }}=\frac{\sum_{j=1}^{n} e_{\text {ave }, \text { trial }, j}}{n}
$$

Twenty trials were recorded using a camera. For each trial, a randomly selected twenty-second segment was used. From the beginning to the end of the clip (from 0 to 20 seconds), an image was taken every 0.2 seconds for the warning test. The total number of such twenty-second trials, $n$, was set to 20 .

Table 1 shows the values of the average position error for each trial obtained using Eq. (21). The distribution of position error for the 20 trials is shown in Fig. 13, where the $x$-axis is the screenshots of each trial, the $y$-axis is the trial number (trials 1 20), and the $z$-axis is the value of the position error. Using Eq. (23), the average position error for the 20 trials is obtained as $0.6509 \mathrm{~cm}$. Compared to the dimensions of an obstacle seen by the driver through the windshield, often about $5 \mathrm{~cm} \times 5 \mathrm{~cm}$, the position error is acceptable.

Table 1 Average position error for 20 trials.

\begin{tabular}{c|c|c|c}
\hline \hline Trial & $e_{\text {ave }, \text { trial }}(\mathbf{c m})$ & Trial & $e_{\text {ave,trial }}(\mathbf{c m})$ \\
\hline 1 & 0.6004 & 11 & 0.4865 \\
2 & 0.6106 & 12 & 0.5406 \\
3 & 0.5031 & 13 & 0.5758 \\
4 & 0.6390 & 14 & 0.6074 \\
5 & 0.7103 & 15 & 0.6185 \\
6 & 0.5617 & 16 & 0.6139 \\
7 & 0.5715 & 17 & 0.6634 \\
8 & 0.6452 & 18 & 0.6892 \\
9 & 0.6266 & 19 & 0.7445 \\
10 & 0.4774 & 20 & 0.6327 \\
\hline \multicolumn{2}{c}{$\sum_{j=1}^{20} e_{\text {ave,trial }, j}$} \\
\hline
\end{tabular}

\section{Conclusion}

This paper presents the design, the prototyping, and the experimental implementation of a windshield warning system. The system indicates a potential hazardous obstacle on the windshield using a laser mark produced by a two-dimensional laser projector unit, allowing the driver to keep attention on the road. An obstacle in front of the vehicle is recognized using cameras and image processing techniques. The coordinates of the obstacle relative to the moving vehicle are calculated and then the line-of-sight equation between the driver and the obstacle is constructed in real-time. The intersection point of the line of sight and the windshield is determined and then a laser projector emits a laser beam to form a warning mark at that point. Both the hardware and the software of the windshield warning system were successfully integrally implemented on a commercial vehicle (Luxgen ${ }^{\circledR}$ MPV) to demonstrate its feasibility. From the 20 trials of the in-vehicle experiments, the average position error of the warning mark was $0.6059 \mathrm{~cm}$, which is only a few percents of the size 
of the obstacle viewed through the windshield. The verification of the proposed system over long driving distances on the streets will be conducted in future work.

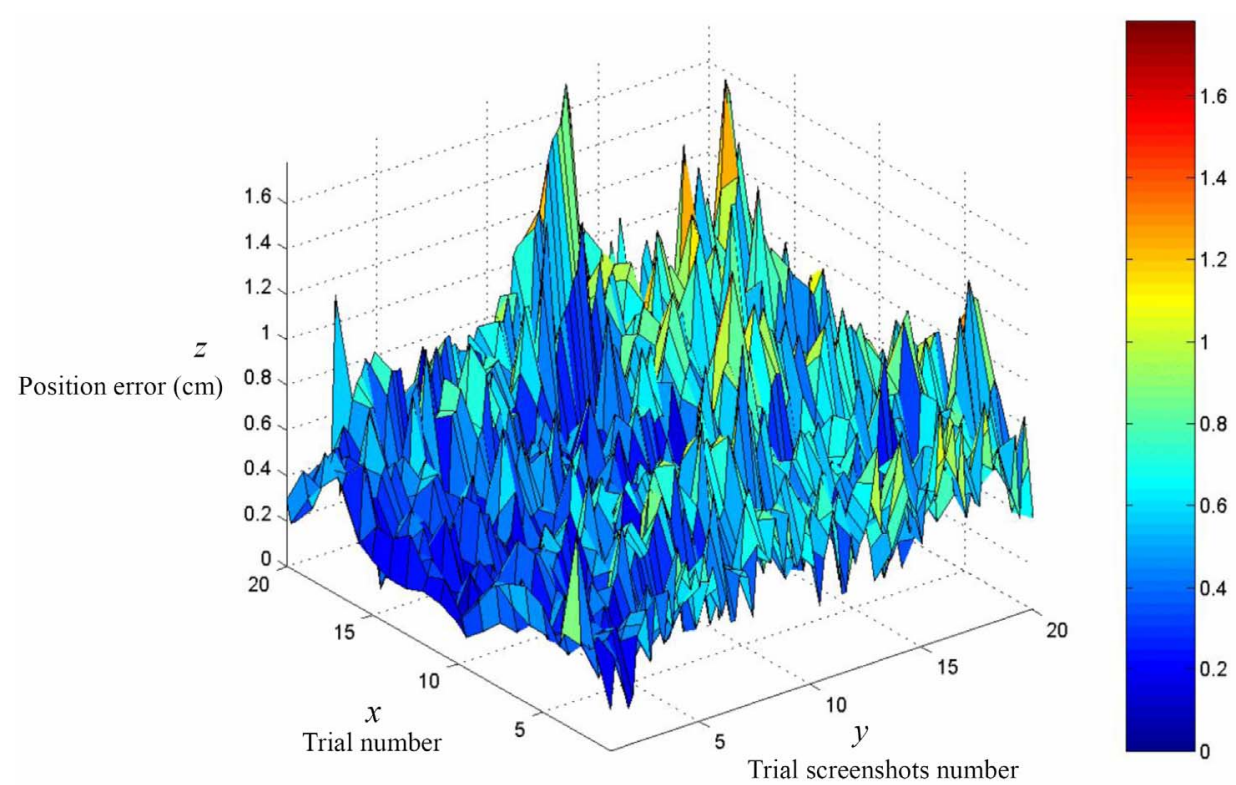

Fig. 13. Distribution of position error for 20 trials.

\section{References}

(1) Curio, C., Edelbrunner, J., Kalinke T., Tzomakas, C., and Seelen, W. V., Walking Pedestrian Recognition, IEEE Transactions on intelligent transportation system, Vol. 1, (1999), pp. 87-93.

(2) Stiller, C., Hipp, J., Rössig, C., and Ewald, A., Multisensor obstacle detection and tracking, Journal of Image and Vision Computing, Vol. 18, (2000), pp. 389-396.

(3) Bensrhair, A., Bertozzi, M., Broggi, A., Miché, P., Mousset, S., and Toulminet, G., A Cooperative Approach to Vision-Based Vehicle Detection, Proceedings of the 2001 IEEE Intelligent Transportation System, (2001), pp. 207-212.

(4) Hsieh, W. C., Fu, L. C., and Huang, S. S., Vision Based Obstacle Warning System for On-Road Driving, Proceedings of the 2003 IEEE International Conference on Intelligent Robots and Systems, (2003), pp. 3668-3673.

(5) Kastrinaki, V., Zervakis, M., and Kalaitzakis, K., A Survey of Video Processing Techniques for Traffic Applications, Journal of Image and Vision Computing, (2003), pp. 359-381.

(6) Huang, S. S., Chen, C. J., Hsiao, P. Y., and Fu, L. C., On-Board Vision System for Lane Recognition and Front-Vehicle Detection to Enhance Driver's Awareness, Proceedings of the 2004 IEEE International Conference on Robotics \& Automation, (2004), pp. 2456-2461.

(7) Li, X., Yao, X., Murphey, Y. L., Karlsen, R., and Gerhart, G., A Real-Time Vehicle Detection and Tracking System in Outdoor Traffic Scenes, Proceedings of the $17^{\text {th }}$ International Conference on Pattern Recognition, Vol. 2, (2004), pp. 761-764.

(8) McCall, J. C. and Trivedi, M. M., Video-Based Lane Estimation and Tracking for Driver Assistance: Survey, System, and Evaluation, IEEE Transactions on Intelligent transportation systems, Vol. 7, No. 1 (2006), pp. 20-37.

(9) Wei, Y., Meng, H., Zhang, H., and Wang, X., Vehicle Frontal Collision Warning System Based on Improved Target Tracking and Threat Asseaament, Proceedings of the 2007 IEEE Intelligent Transportation System, (2007), pp. 167-172.

(10) Sengupta, R., Rezaei, S., Shladover, S. E., Cody, D., Dickey, S., and Kariharan, K., 
Cooperative Collision Warning Systems: Concept Definition and Experimental Implementation, Journal of Intelligent Transportation Systems, Vol. 11, No. 3 (2007), pp. 143-155.

(11) Jenkins, D. P., Stanton, N. A., Walker, G. H., and Young, M. S., A New Approach to designing lateral collision warning system, International Journal of Vehicle Design, Vol. 45, No. 3 (2007), pp. 379-396.

(12) Hammoud, R. I., Smith, M. R., Dufour, R., Bakowski, D., and Witt, G., Driver Distraction Monitoring and Adaptive Safety Warning Systems, Proceedings of SAE Commercial Vehicles Engineering Congress and Exhibition, (2008), Paper No. 2008-01-2694.

(13) Kim, S. Y., Kang, J. K., Oh, S. Y., Ryu, Y. W., Kim, K., Park, S. C., and Kim J., An Intelligent and Integrated Driver Assistance System for Increased Safety and Convenience Based on All-Around Sensing, Journal of intelligent Robot Systems, (2008), pp. 261-287.

(14) Gao, D., Li, W., Duan, J., and Zheng, B., A Practical Method of Road Detection for Intelligent Vehicle, Proceedings of the 2009 IEEE International Conference on Automation and Logistics, (2009), pp. 980-985.

(15) Hsiao, P. Y., Yeh, C. W., Huang, S. S., and Fu, L. C., A Portable Vision-Based Real-Time Lane Departure Warning System: Day and Night, IEEE Transactions on Vehicular Technology, Vol. 58, No. 4 (2009), pp. 2089-2094.

(16) Lin, C. W., Wang, H. Y., and Tseng, D. C., A Robust Lane Detection and Verification Method for Intelligent Vehicles, Third International Symposium on Intelligent Information Technology Application, Vol. 1, (2009), pp. 521-524.

(17) Lin, Y. R., and Li, Y. H., FPGA Implementation of a Vision-Based Blind Spot Warning System, World Academy of Science, Engineering and Technology, (2010), pp. 896-900.

(18) Flores, M. J., Armingol, J. M., and Escalera, A. D. L., Real-Time Warning System for Driver Drowsiness Detection Using Visual Information, Journal of Intelligent and Robotic Systems, Vol. 59, (2010), pp. 103-125.

(19) Dahmani, H., Chadli, M., Rabhi, A., and Hajjaji, A. El., Driver Attention Warning System Based on a Fuzzy Representation of the Vehicle Model, Proceedings of the $18^{\text {th }}$ International Federation of Automatic Control, (2011), pp. 6260-6265.

(20) Gellert, W., Gottwald, S., Hellwich, M., Kästner, H., and Künstner, H., Plane, (1989), pp. 539-543, VNR Concise Encyclopedia of Mathematics, New York.

(21) Algebraic plane. (online), available from $<$ http://mathworld.wolfram.com/Plane.html $>$, (accessed 2012-02-22).

(22) Quest 3D software. (online), available from $<$ http://www.quest3d.com/>, (accessed 2012-02-22).

(23) Servo motor. (online), available from $<$ http://www.servodatabase.com/servo/futaba/s3102>, (accessed 2012-02-22).

(24) SSC-32 servo controller. (online), available from $<$ http://www.lynxmotion.com/p-395-ssc-32-servo-controller.aspx $>$, (accessed 2012-02-22). 